\title{
Análisis estructural del cigüeñal de un motor MWM sprint 2.8 I en autodesk inventor
}

\section{Structural analysis of the crankshaft of an MWM sprint $2.8 \mathrm{l}$ engine in autodesk inventor}

Ing. Fredy Leonardo Morquecho Andrade, MSc. Universidad Internacional del Ecuador, Ecuador

Autor para correspondencia: frmorquechoan @internacional.edu.ec

Fecha de recepción: 01 de octubre de 2018 - Fecha de aceptación: 15 noviembre de 2018

\section{Resumen}

El presente estudio se enfoca en un análisis estructural del cigüeñal de un motor MWM sprint 2.8 L en autodesk inventor. En la parte metodológica se revisó documentos bibliográficos relacionados con este tema con el fin de obtener datos correctos para determinar los esfuerzos a los que está sometido el cigüeñal; tales como: esfuerzos de flexión y torsión. Se realizarán simulaciones donde se apliquen presiones en puntos específicos, usando para ello un método de elementos finitos. Los resultados que se muestran en las conclusiones sobre el análisis de los esfuerzos del cigüeñal se ven alterado por una serie de parámetros tales como; la relación de compresión, cilindrada, presión en el cilindro, dimensiones de biela y pistón, entre otros.

Palabras clave: elementos finitos; nodos; esfuerzo de flexión; esfuerzo de tensión; cigüeñal; motor

\begin{abstract}
This study focuses in the structural analysis of the crankshaft in a 2.8L MWM Sprint engine using Autodesk Inventor. The methodology of the study involved an extensive literature review of studies related to the topic with the purpose of obtaining accurate data to determine the effort to which the crankshaft is subjected, e.g. flexion and torsion. Simulations will be performed applying pressure on specific points, implementing the method of finite elements. The results shown in the conclusions about the analysis of the crankshaft endurance are influenced by a series of factors as the compression relation, displacement, cylinder pressure, connecting rod, and piston dimensions, among others.
\end{abstract}

Key words: finite elements; nodes; flexion effort; tension effort; crankshaft; engine 


\section{Introducción}

El cigüeñal es el componente largo con una geometría compleja dentro del motor, el cual convierte el desplazamiento lineal del pistón en rotativo. A lo largo del funcionamiento del cigüeñal está sometido a un sin número de cargas durante todo el ciclo de vida de ese motor, es por esto que la durabilidad de los componentes y los esfuerzos que soporta el cigüeñal deben ser consideradas en el proceso de diseño de esta parte del motor (Valdés, Coronado, \& García, 2006).

Para el desarrollo de este proyecto se usa como herramienta el programa Inventor de Autodesk, prácticamente se basa en técnicas de software CAD paramétrico. Permite comenzar de un diseño de componentes que se pueden combinar en ensamblajes, obteniendo diversas variantes.

Un modelador paramétrico permite modelar la geometría, dimensión y material de manera que, si se alteran las dimensiones, la geometría se actualiza automáticamente tomando las nuevas dimensiones. Esto permite que el diseñador almacene sus conocimientos de cálculo dentro del modelo, a diferencia del modelado no paramétrico, que está más relacionado con un "tablero de bocetos digitales". Inventor también tiene herramientas para la creación de piezas metálicas. Cuenta también con análisis de tensiones por elementos finitos y análisis dinámicos. Creación y análisis de estructuras, piping y cableado, y las tecnologías iPart, iAssembly, iMates, iCopy, iLogic hacen que el diseño sea más rápido y eficiente. (Mecánica y Control para Industriales, 2010)

Este es uno del software más completo, por lo que es considerado para el desarrollo de este proyecto, en base a los datos obtenidos mediante las fórmulas de fuerzas que se generan en un motor de combustión, estos datos servirán para ubicarlos en la barra de tareas de Inventor. De esta manera se podrán observar cuales son los puntos del cigüeñal donde se generan más esfuerzos.

\section{Propiedades de los materiales}

Los materiales que se utilizan en la construcción de cigüeñales son variados, y esto se debe principalmente al tamaño y factores de velocidad y potencia del motor. Por estas razones para que el cigüeñal tenga un buen rendimiento se deben tener en cuenta los siguientes lineamientos:

Un límite elástico el cual no perjudique su funcionamiento, ni los demás elementos del motor, un coeficiente de resistencia tal que soporte las cargas de combustión, el límite de fatiga y coeficiente de amortiguamiento para reducir las vibraciones en el motor.

El coeficiente de fricción debe ser pequeño para evitar un desgaste excesivo, debe tener un proceso de manufacturado, en cual su acabado no se vea afectado por porosidad, que afecten a su rendimiento. 
Generalmente los cigüeñales están fabricados de acero forjado o laminado (aceros al carbono) y sus dimensiones dependen exclusivamente de los esfuerzos y vibraciones a los que está sometido, según el trabajo, generalmente los cigüeñales están sometidos a un gran número de ciclos de trabajo durante la vida útil del motor. Los mismos deben ser diseñados cuidadosamente para conseguir el menor peso y dimensiones posibles. Debido a esta razón, para el proceso de diseño se debe evaluar los comportamientos ante posible fatiga, eficiencia energética, debido a las exigentes restricciones a nivel estructural de un cigüeñal, en su diseño debe garantizarse el cumplimiento de su equilibrio estático, distribuyendo todo su peso de forma uniforme alrededor de las bancadas y equilibrio dinámico para eliminar cualquier vibración que pueda generarse durante el giro por causas que ejercen las bielas. (Norton, 2009)

\section{Perplejidad en los cigüeñales}

Las fuerzas alterna y centrífuga de los órganos en movimiento y las presiones del gas, dan origen a fuerzas y a momentos que actúan sobre la estructura del motor. Debido a que dichas fuerzas y momentos son variables en el tiempo, si no se realiza su equilibrado, aparecerán vibraciones indeseables, que además de hacer más incómoda su utilización, generarán averías por la aparición de fatigas en sus elementos. (Cinemática y Dinámica del motor, 2018)

Según Ortiz (2016), afirma que: "El equilibrio por adición de pesos, que crea fuerzas iguales y opuestas a aquellas de la inercia, es conocido como compensación." Generalmente, después de la compensación todavía hay algunas pequeñas fuerzas remanentes que no han sido completamente equilibradas.

Cuando el cigüeñal se encuentra equilibrado estáticamente la resultante de las fuerzas centrífugas debe ser nula, dando como resultado a que el baricentro se encuentre sobre el eje de rotación. Esto es característico para el cigüeñal con una sola manivela. Para que el cigüeñal esté en equilibrio en cualquier posición, el peso de la manivela debe estar equilibrado con sus respectivas contrapesas.

El cigüeñal además de estar equilibrado estáticamente, puede estarlo dinámicamente, es decir, puede dar lugar, cuando se halla en movimiento, a un momento flector originado por las fuerzas centrífugas que se encuentran en planos distintos. Puede suceder, en definitiva, que en los motores con varios cilindros la resultante de los momentos respecto a un punto cualquiera del eje no sea nula. Las fuerzas centrífugas actúan en planos diferentes, cuya distancia entre ellos es igual a la que hay entre los ejes de los cilindros. El momento resultante es igual al producto de la fuerza centrífuga por la distancia entre los ejes y puede ser equilibrado con uno igual y opuesto por medio de contrapesos, como se muestra en la figura 1 (Sánchez, 2016)

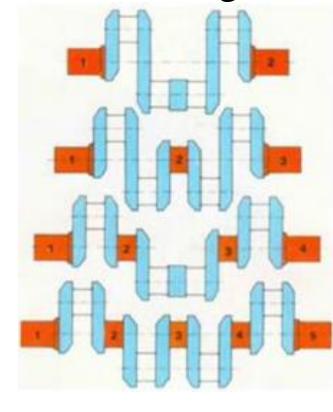


Figura 1. Cigüeñal equilibrado estáticamente (Sánchez, 2016)

En cuanto al equilibrio dinámico, los cigüeñales con más de dos manivelas resultan equilibrados cuando estando ya compensados estáticamente, admiten un plano de simetría perpendicular al eje de rotación respecto al cual las manivelas resultan simétricas en número, posición y dimensiones. Algunos diseños de cigüeñales aun estando en conjunto equilibrados dinámicamente, incluso sin contrapesos, tienen equilibradas cada una de las manivelas por separado. Este tipo de contrapesado sirve para reducir la carga impuesta a los cojinetes de bancada

Efectivamente, contrapesando cada una de las manivelas se reducen, o se anulan, los diversos momentos flectores, debido a las masas giratorias que, actuando sobre las diferentes partes del cigüeñal. (Arrieta, 2011)

Existen cigüeñales, a los cuales se les debe tener en conjunto un equilibrio dinámico, incluso sin sus respectivos contrapesos, teniendo un equilibrio cada una de las manivelas por separado. Este tipo de contrapesado sirve para reducir la carga impuesta a los cojinetes de bancada. Equilibrando cada manivela se reducen, o se anulan, diversos momentos flectores, por sus masas giratorias que, actúan sobre las diferentes partes del cigüeñal. En la figura 2 se observa un equilibrado estático referente a las masas de un motor monocilíndrico, y en la siguiente (figura 3), el equilibrado dinámico de las masas en rotación de un motor bicilíndrico.

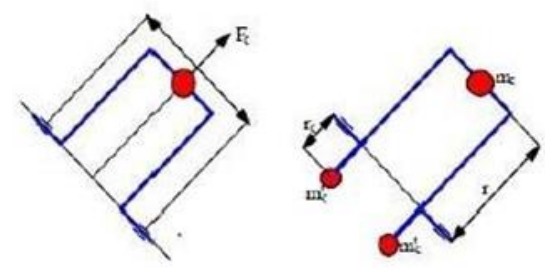

Figura 2. Equilibrado estático en un cigüeñal monocilindrico (Sánchez, 2016)

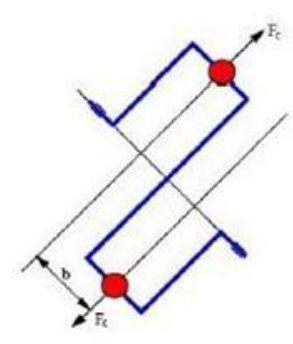

Figura 3. Equilibrado dinámico de un cigüeñal bicilindrico (Sánchez, 2016)

\section{Elementos finitos}

El método de elementos finitos (MEF), es un método de resolución aproximativo, el cual se utiliza principalmente en problemas que involucran un alto grado de complejidad, en los cuales no se puede conocer la solución exacta. Este método ha adquirido con el uso de los ordenadores una gran importancia en la solución de problemas de ingeniería, física. Que permite 
resolver casos que hasta hace poco tiempo eran prácticamente imposibles de resolver por métodos matemáticos tradicionales. Esta circunstancia obligaba a realizar prototipos para ensayos, e ir realizando mejoras de forma iterativa, lo que traía consigo un elevado costo tanto económico como en tiempo de desarrollo (Sánchez D. O., 2016).

Para el análisis de elementos finitos debe existir el dominio, espacio geométrico en cual se va a trabajar la pieza, las condiciones de contorno, que son variables conocidas y que condicionan el cambio del sistema y las variables de como actuaran las fuerzas de fatiga y flexión. (Imperial, 1996).

Fuerzas en el tren alternativo del motor de combustión interna. figura 4 .

Las distintas fuerzas que actúan en las partes alternativas del motor son mostradas en la

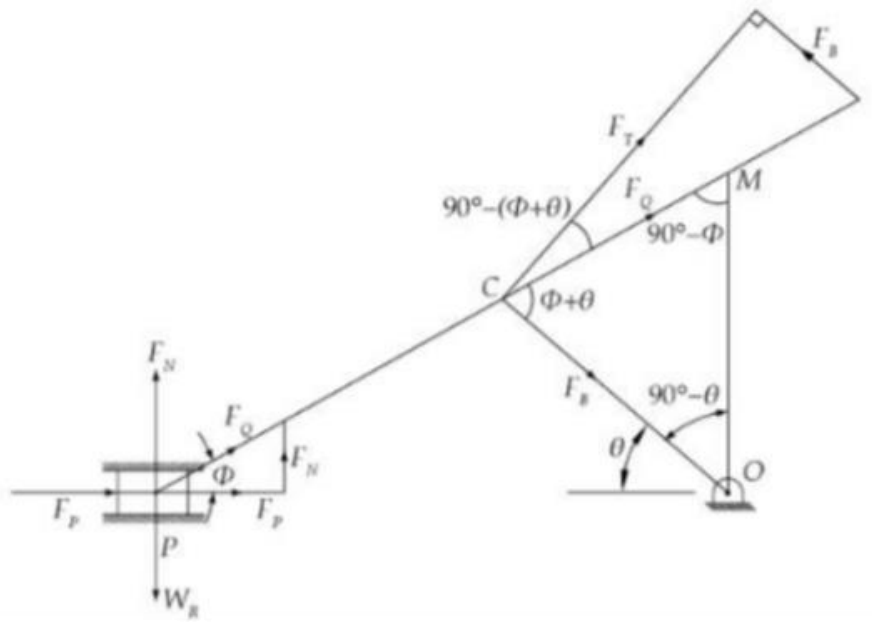

Figura 4. Fuerzas en las partes alternativas (Norton, 2009)

Muchas de las expresiones de estas fuerzas, omitiendo el peso de la biela son derivadas como: esfuerzo del pistón.

La fuerza neta que actúa en el pistón a lo largo de la carrera es llamada esfuerzo del pistón, y es denotado por Fp.

$$
F_{-} P=F_{-} L \pm F_{-} 1-R_{-} f
$$

En el caso de un motor horizontal $F L$ es la carga neta que actúa en el pistón. Para un único cilindro $p \cdot A$ y para una doble acción (p_1 A_1-p_2 A_2) donde $\mathrm{p}, \mathrm{p} \_(1,)$ p_2 representan presiones y A, A_1, A_2 representan el área de la sección transversal del cilindro en cualquier lado del pistón. Por lo tanto, la fuerza o carga neta en el pistón es:

$$
F_{-} L=\left(p_{-} 1-p_{-} 2\right) A_{-} 1
$$

F_I es la fuerza de inercia de las partes alternativas y R_F es la resistencia a la fricción. 
Las fuerzas de inercia F_I debido a la aceleración de las partes alternativas se opone a la fuerza de aceleración $\mathrm{F}_{-} \mathrm{P}$ en el pistón. Usa signo negativo para un ángulo entre $0^{\circ}$ y $180^{\circ}$, y se tiene un signo positivo entre $180^{\circ}$ y $360^{\circ}$.

Usando las expresiones derivadas de las aceleraciones, la fuerza de inercia es dada por:

$$
F_{I}=m_{R} \cdot a_{R}=m_{R} w^{2} r\left\{\cos \theta+\frac{\cos 2 \theta}{n}\right\}
$$

Donde $\mathrm{m} \_\mathrm{R}$ es la masa de las partes alternativas y a_R es la aceleración de las partes alternas.

Donde $\mathrm{r}$ es el radio del cigüeñal y $\theta$ es el ángulo.

$$
F_{P}=\left(F_{L} \pm F_{1} \pm W_{R}-R_{F}\right)
$$

Para un motor vertical, el peso de las partes alternas ayuda al esfuerzo del pistón mientras se mueve hacia abajo y se opone cuando se mueve hacia arriba. Así que se tiene + W_R para el movimiento descendente $\mathrm{y}-\mathrm{W} \_\mathrm{R}$ para el movimiento ascendente.

\section{Fuerza a lo largo de la biela (F_Q)}

$$
F_{Q}=\frac{F_{P}}{\cos \emptyset}=\frac{F_{P}}{\sqrt{\left[1-\frac{\operatorname{sen}^{2} \theta}{\mathrm{n}^{2}}\right]}}
$$

$\theta)$. )

Desde la geometría de la figura y $L \operatorname{sen} \emptyset=\mathrm{r} \operatorname{sen} \emptyset$ o $\operatorname{sen} \emptyset=(\operatorname{sen} \emptyset) / n ; \cos \emptyset=\sqrt{ }\left(\left(1-\operatorname{sen}^{\wedge} 2\right.\right.$

Por lo tanto:

$$
\cos \emptyset=\sqrt{\left[1-\frac{\operatorname{sen}^{2} \theta}{n^{2}}\right]}
$$

Empuje en los lados del cilindro (FN):

$$
\begin{aligned}
& F_{N}=F_{Q} \operatorname{sen} \emptyset=\frac{F_{P} \operatorname{sen} \emptyset}{\cos \emptyset} \\
& F_{Q}=\frac{F_{P}}{\cos \emptyset} \\
& F_{N}=F_{P} \tan \emptyset=\frac{F_{P} \operatorname{sen} \emptyset}{\sqrt{\left[1-\frac{\operatorname{sen}^{2} \theta}{n^{2}}\right]}}
\end{aligned}
$$




\section{Esfuerzos en los apoyos del cigüeñal $\left(F_{-} T\right)$ y empuje en los cojinetes del cigüeñal}

El componente F_Q perpendicular al cigüeñal es llamada esfuerzo del apoyo indicado por F_T y el componente a lo largo del cigüeñal el cual produce empuje a los cojinetes es F_B.

$$
\begin{aligned}
& F_{T}=F_{Q} \operatorname{sen}(\theta+\emptyset)=\frac{F_{P} \operatorname{sen}(\theta+\emptyset)}{\cos \emptyset} \cdot r \\
& F_{B}=F_{Q} \cos (\theta+\emptyset)=\frac{F_{P} \cos (\theta+\emptyset)}{\cos \emptyset}
\end{aligned}
$$

\section{Esfuerzo torsional en el cigüeñal}

El producto del esfuerzo en los apoyos $\left(\mathrm{F}_{-} \mathrm{T}\right)$ y el radio del cigüeñal son conocidos como esfuerzo del cigüeñal o momento torsional.

$$
\begin{gathered}
\quad T=F_{T} \cdot r=\frac{F_{P} \operatorname{sen}(\theta+\emptyset) \cdot \mathrm{r}}{\cos \emptyset} \\
=F_{P} \frac{(\operatorname{sen} \theta \cos \emptyset+\cos \emptyset \operatorname{sen} \theta) \cdot \mathrm{r}}{\cos \emptyset} \\
T=F_{P}(\operatorname{sen} \theta+\cos \theta \cdot \tan \emptyset) \cdot r \\
\text { Por lo tanto, } \tan \emptyset=\frac{\operatorname{sen} \theta}{\sqrt{n^{2}}-\operatorname{sen}^{2} \theta}
\end{gathered}
$$

\section{El esfuerzo en el cigüeñal o torque}

$$
T=F_{P} r\left[\operatorname{sen} \theta+\frac{\operatorname{sen} 2 \theta}{\left(2 \sqrt{\mathrm{n} 2}-\operatorname{sen}^{2} \theta\right)}\right]
$$

Por lo tanto sen $2 \Theta$ es muy pequeño comparado a $n 2$, así que sen $2 \theta$ se desprecia $u$ omite.

$$
\begin{aligned}
& T=F_{P}\left[\operatorname{sen} \theta+\frac{\operatorname{sen} 2 \theta}{2 n}\right]=F_{P} \cdot O M \\
& \text { Donde } O M=r\left[\operatorname{sen} \theta+\frac{\operatorname{sen} 2 \theta}{2 n}\right]
\end{aligned}
$$

\section{Cálculo de esfuerzos en un motor MWM SPRINT $2.8 \mathrm{~L}$}

Datos

$\mathrm{n}=1500 \mathrm{rpm}$

$\mathrm{s}=103 \mathrm{~mm}$

$\mathrm{Vu}=700 \mathrm{~cm} 3$

R_c $=17,2: 1$

a_p $=1206072 \mathrm{~m} / \mathrm{s}^{\wedge} 2$ (Aceleración lineal del pistón)

$\mathrm{r}=57.35 \mathrm{~mm}=0.05735 \mathrm{~m}$ (Radio de giro del cigüeñal) 
R_F $=250 \mathrm{~N}$ (Fricción existente entre el cilindro y pistón)

$\mathrm{mR}=22.732 \mathrm{~kg}$ (Masa del cigüeñal y pistón)

$\theta=45^{\circ}$ (Ángulo de desplazamiento del cigüieñal)

$\mathrm{p}=1,72 \mathrm{~N} / \llbracket \mathrm{mm} \rrbracket \wedge 2$ (Presión efectiva en el cilindro)

$\mathrm{D}=110 \mathrm{~mm}=0.11 \mathrm{~m}$ (Diámetro del pistón)

$\mathrm{N}=\mathrm{L} / \mathrm{r}=0.05 / 0.015=3.33$ (Relación entre centros; ejes de biela y cigüeñal)

\section{Presión efectiva en el pistón}

Primero se debe conocer la presión que se genera en el cilindro, aquella presión es la que se transmite al pistón y posteriormente a las demás partes alternativas. Con el dato de la relación de compresión, y la cilindrada unitaria, se es posible conocer la presión generada.

$$
\begin{aligned}
& \frac{P_{1}}{V_{2}}=\frac{V_{2}}{V_{1}} ; P_{1} \cdot V_{1}=P_{2} \cdot V_{2} \\
& P_{1}=1 \mathrm{bar} \text { (Aproximadamente a la atmosférica) } \\
& V u=700 \mathrm{~cm} 3 \text { (Cilindrada unitaria) }
\end{aligned}
$$

Como la relación de compresión es de 17,2:1, indica que el volumen en el cilindro se ha reducido 17,2 veces; Por lo tanto:

$$
\begin{aligned}
& V_{2}=\frac{700 \mathrm{~cm}^{3}}{17,2}=40,69 \mathrm{~cm}^{3} \\
& \text { Reemplazando; } \\
& P_{2}=\frac{P_{1} \cdot V_{1}}{V_{2}}=\frac{1 \mathrm{bar} .700 \mathrm{~cm}^{3}}{40.69 \mathrm{~cm}^{3}} \\
& P_{2}=17.20 \mathrm{bar}
\end{aligned}
$$

La presión que se genera en el cilindro es de 17,20 bars, cuya cifra es equivalente a $1,72 \mathrm{~N} / \llbracket \mathrm{mm} \rrbracket \wedge 2$

\section{Aceleración lineal del pistón}

$$
\begin{aligned}
& \alpha=\frac{n^{2} \cdot s}{182400}\left(\cos \varphi+\frac{r}{l} \cos 2 \varphi\right) \\
& \alpha=\frac{1500^{2} \cdot 103 \mathrm{~mm}}{182400}\left(\cos 45^{\circ}+\frac{57.35}{15} \cos 90^{\circ}\right) \\
& \alpha=898.42 \frac{\mathrm{m}}{\mathrm{s}^{2}}
\end{aligned}
$$

Esfuerzo del pistón $\left(F_{-}\right.$P $)=F_{-} L_{-}-F_{-} I$ 


$$
\begin{aligned}
& F_{L}=\frac{p \pi d^{2}}{4} \\
& =\frac{1,72 \pi(110)^{2}}{4}=16345 \mathrm{~N}=16.34 \mathrm{KN} \\
& F_{I}=m_{R} \cdot a_{P} \\
& F_{I}=22.732 \cdot 898.42 \mathrm{~m} / \mathrm{s}^{2} \\
& =20422 \mathrm{~N}=20.42 \mathrm{KN} \\
& \left(F_{P}\right)=F_{L}-F_{I}-R_{F} \\
& \left(F_{P}\right)=20422-16345-22.732 \\
& =4054.26 \mathrm{~N}=4.05 \mathrm{KN}
\end{aligned}
$$

\section{Presión en el conjunto pistón-biela}

$$
\begin{aligned}
& F_{N}=F_{P} \tan \varnothing \\
& \text { Donde } \operatorname{sen} \emptyset=\frac{\operatorname{sen} \varnothing}{n}=\frac{\operatorname{sen} 45^{\circ}}{4}=\frac{0.707}{4}=0.176 \\
& \emptyset=10.13^{\circ} \\
& \text { Por lo tanto; } \\
& F_{N}=4054.26 \cdot \tan 10.13^{\circ}=724.36 \mathrm{~N}
\end{aligned}
$$

\section{Empuje en los cojinetes del cigüeñal}

$$
\begin{aligned}
& F_{B}=\frac{F_{P} \cos (\theta+\emptyset)}{\cos \emptyset} \\
& =\frac{4054.26 \cos \left(45^{\circ}+10.13^{\circ}\right)}{\cos 10.13^{\circ}} \\
& =\frac{4054.26 \cdot 0.57}{0.984} \\
& =2348.50 \mathrm{~N}=2.34 \mathrm{KN}
\end{aligned}
$$

\section{Esfuerzo torsional en el cigüeñal}




$$
\begin{aligned}
& T=F_{T} \cdot r \\
& =\frac{F_{P \operatorname{sen}(\theta+\emptyset) \cdot r}}{\cos \emptyset} \\
& =\frac{4054.26 \cdot \operatorname{sen}\left(45^{\circ}+10.13^{\circ}\right) \cdot 0.05735}{\cos 10.13^{\circ}} \\
& =\frac{4054.26 \cdot 0.820 \cdot 0.05735}{\cos 10.13^{\circ}} \\
& =\frac{4054.26 \cdot 0.820 \cdot 0.05735}{0.984} \\
& =193.78 \mathrm{~N}=0.19 \mathrm{KN}
\end{aligned}
$$

\section{Materiales y métodos}

En este apartado se realiza el diseño de cada una de las piezas que componen el cigüeñal.

La elaboración de la simulación de esfuerzos en el cigüeñal, está basada en información técnica, la cual se obtuvo del manual de taller (Workshop Manual) del motor MWM Sprint, aunque la pieza de estudio en el proyecto es el cigüeñal, deben conocerse ciertas características del motor o las demás piezas del tren alternativo ya que las mismos trabajan en conjunto con el cigüeñal por lo tanto, las especificaciones de cilindrada, relación de compresión, dimensiones del pistón y biela se tomaron del manual de taller, aquellos datos son útiles para el cálculo de esfuerzos.

El software utilizado, es Autodesk Inventor Professional, este programa, permite diseñar cuerpos sólidos y realizar simulaciones. Utilizando el programa se diseña el ciguieñal, con las dimensiones que indica el manual, en la simulación es necesario conocer los esfuerzos a los que está sometido y cuáles son los valores de dichos esfuerzos, estos datos se los obtienen realizando las ecuaciones analizadas anteriormente, en la figura 5 se puede ver las dimensiones del cigüeñal MWM sprint (K. Thriveni, Dr.B.Jaya Chandraiah, 2013). 


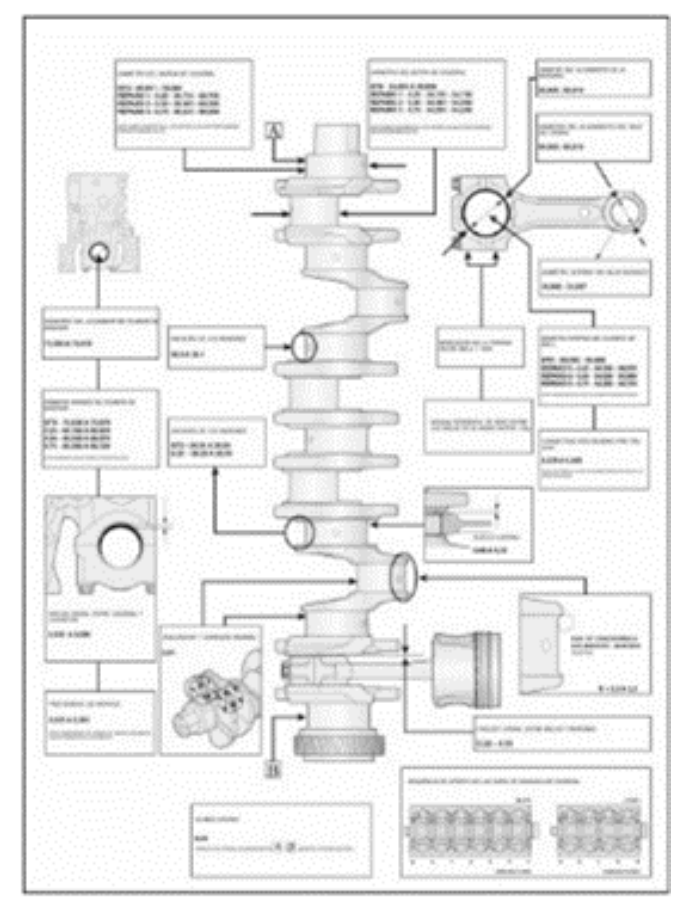

Figura 5. Dimensiones del cigüeñal MWM Sprint (Manual de Taller)

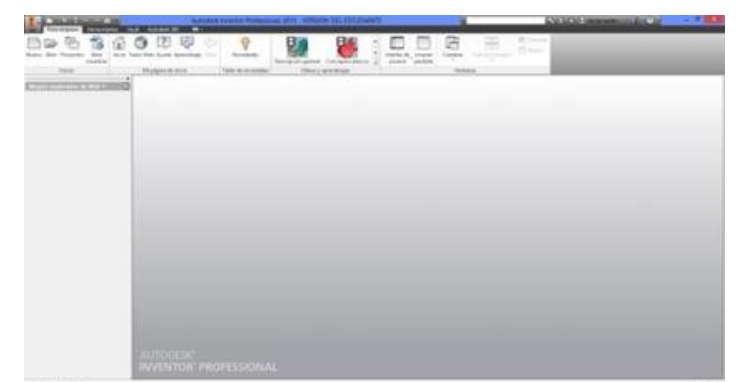

Figura 6. Software de diseño y simulación (Propia)

Se mostrará los elementos realizados en Inventor para el diseño del cigüeñal, usada para los elementos finitos.

La geometría estructural está compuesta por la nariz, muñequillas, contrapesos, arandelas, brida, el conjunto delimitará un único volumen, el cual será tratado como un sólido elástico y lineal para el cual se calcularán las tensiones, deformaciones y desplazamientos, en las siguientes figuras se puede ver los componentes de la estructura del cigüeñal.

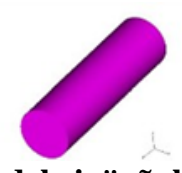

Figura 7. Nariz del cigüeñal (Sánchez, 2016) 


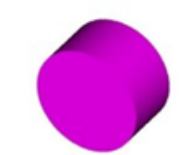

Figura 8. Geometría de la bancada del cigüeñal (Sánchez, 2016)

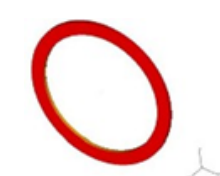

Figura 9. Arandela del cigüeñal (Sánchez, 2016)

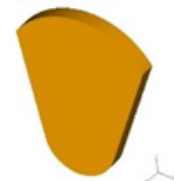

Figura 10. Contrapeso del cigüeñal (Sánchez, 2016)

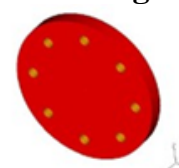

Figura 11. Brida del cigüeñal (Sánchez, 2016)

Una vez realizado cada elemento se procede a la unión de todos los elementos que conforman el cigüeñal, como se puede observar en la figura 12.

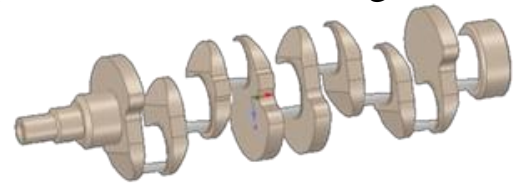

Figura 12. Geometría del cigüeñal (Sánchez, 2016)

Es necesario expresar las condiciones de contorno que afectan al modelo de elementos finitos, si no se especifican de forma adecuada las restricciones o las condiciones de soporte del cigüeñal, el sistema tendrá la libertad de moverse como un cuerpo rígido y no ofrecerá resistencia a las cargas aplicadas.

En la simulación del cigüeñal de un motor de combustión de cuatro tiempos estacionario, se tendrán en cuenta las condiciones a las que es sometido cuando se encuentra en funcionamiento.

\section{Resultados}

En la figura 13 se puede observar los esfuerzos torsionales que reciben los muñones de biela. 


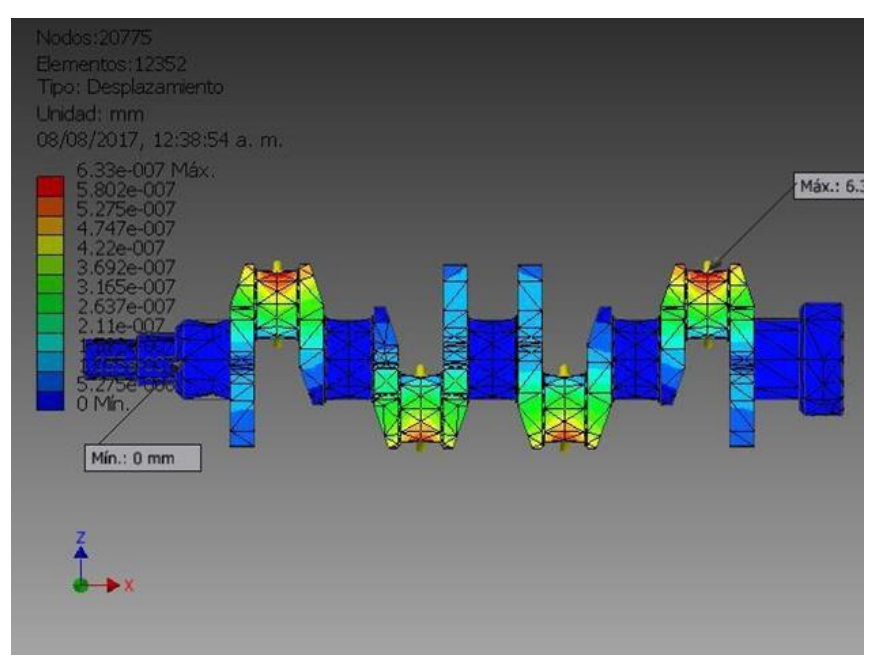

Figura 13. Esfuerzo torsional en los muñones de biela (Propia)

Los cuales provocan una pequeña deformación en aquellas zonas, el color próximo al azul indica un nivel de deformación pequeño mientras que los valores próximos al color rojo indican una deformación relativamente grande. La indicación de la deformación se observa en la barra, aquellos valores están denotados por la notación e, se observa en la figura 14.

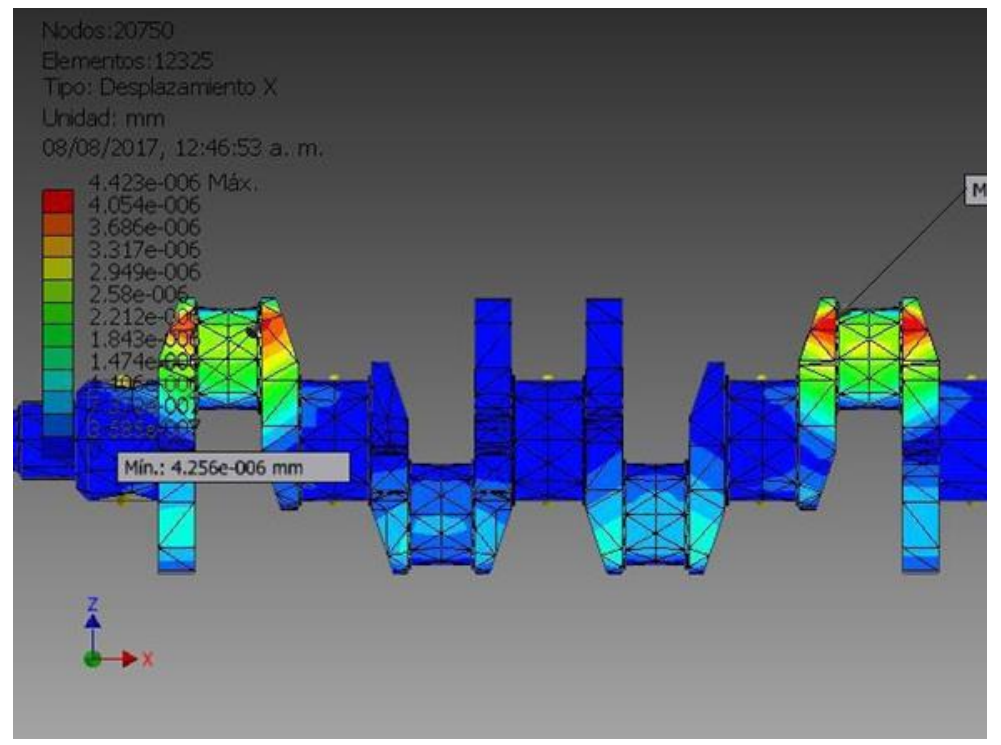

Figura 14. Esfuerzo torsional en los apoyos del cigüeñal (Propia)

En la figura 15 se observa las fuerzas aplicadas a los muñones de biela. 


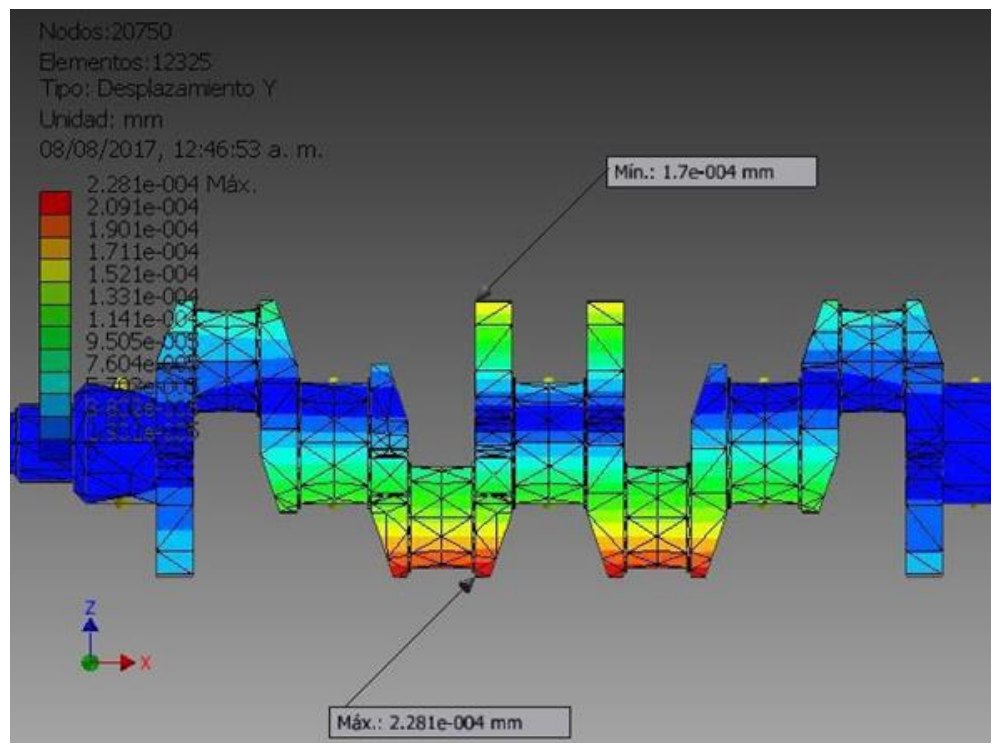

Figura 15. Fuerza aplicada al muñón de biela (Propia)

Parte de los esfuerzos que se transmiten al cigüeñal también son transmitidos a los apoyos, aquellos también reciben un grado de deformación en unidades de milímetros.

La fuerza que se ejerce en el área de los muñones de biela provoca pequeñas deformaciones, las cuales no superan el límite o el grado de deformación plástica del cigüeñal, la lectura de la máxima deformación es 1.801e-004 la cual equivale a 1.801x10-4 mm esa deformación se ubica en los centros de los muñones.

Se toma un ángulo de $45^{\circ}$, referencia de un cigüeñal que posee una fisura, la primera de ellas formando $45^{\circ}$ con el eje del cigüeñal (inclinación que es considerable bajo sobrecarga a torsión) y una segunda que se obtiene mediante la selección del plano que une las zonas con un radio de acuerdo con la muñequilla y la zona de apoyo, como se ve en la figura 16

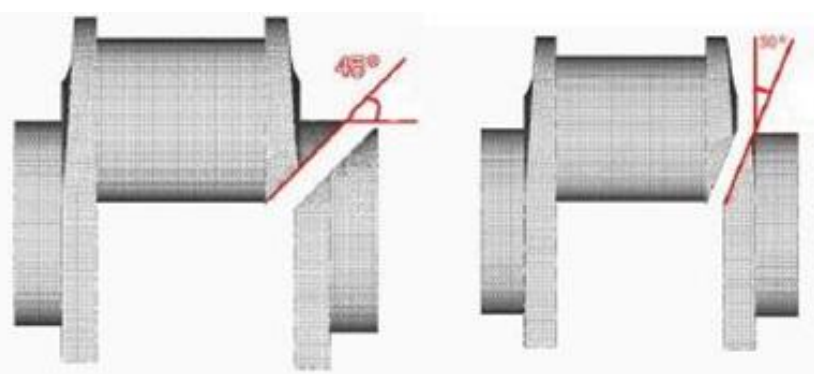

Figura 16. Planos de crecimiento tomados para el estudio (Sánchez, 2016)

\section{Discusión y Resultados}

Para, K. Thirveni (2010) en su estudio: 
"Se usaron determinados desplazamientos, esfuerzo, deformaciones, etc. Debajo de las condiciones de la carga estática en el cuerpo y análisis estático lineal. Linealidades incluidas plásticas, esfuerzos de flexión, deflexión, equilibrado, ultra elasticidad, superficies en contacto.

Son parámetros que se usaron para su estudio de esfuerzos del cigüeñal, cada característica fue aplicada según datos del cigüeñal creados.

En este proyecto fue similar pues se tomaron en cuenta los parámetros de carga estática en el cuerpo del cigüeñal, comparando ambos estudios, se observó que la máxima deformación aparece en el centro del cigüeñal y en los codos del cigüeñal.

Según David Ortiz (2016), concluye que:

"Plantea el análisis del comportamiento resistente del cigüeñal de un motor de combustión de cuatro tiempos. Dicho análisis se lleva a cabo mediante el empleo del Método de Elementos Finitos (MEF).”

Por un lado, se ha llevado a cabo un análisis elástico lineal en el cuál los desplazamientos y tensiones que se producen se consideran admisibles. Se ha estimado un coeficiente de seguridad de 33, estableciendo así un amplio margen de operación.

De acuerdo con el análisis de cada formula y el estudio de las simulaciones realizadas en Inventor. Las cargas afectan al cigüeñal en los muñones de apoyo principal, donde puede llegar a ver fisuras en el caso que no se llegue a tener un buen equilibrado o el cigüeñal no haya sido diseñado para trabajar a altos esfuerzos. Otro dato que se debe tener en cuenta es los componentes que son utilizados para la fabricación del cigüeñal, dependiendo del material tendremos un alto coeficiente de elasticidad, fricción, de estos dependen de que soporte una elevada temperatura, esfuerzos de tensión y flexión.

\section{Conclusiones}

El análisis de los esfuerzos del cigüeñal se ve alterado por una serie de parámetros tales como; la relación de compresión, cilindrada, presión en el cilindro, dimensiones de biela y pistón, entre otros.

Aunque en este proyecto se expresa valores fijos, solo lo hace para parámetros exactos tales como el rpm en las que se encuentra el motor o el ángulo al que se encuentra desplazado el cigüeñal, de manera que a medida que cambian cada uno de estos parámetros se obtienen una gran variedad de valores en esfuerzos.

\section{Bibliografía}

Angelfire. (2018). Obtenido de http://www.angelfire.com/nd/mingopage/ciguenal.htm

Arrieta, K. (28 de 11 de 2011). Kevin Arrieta. Recuperado el 11 de 12 de 2017, de Kevin Arrieta: http://arrieta94.blogspot.com/2011/11/ciguenal.html 
Cinemática y Dinámica del motor. (29 de enero de 2018). Recuperado el 11 de 12 de 2017, de Cinemática y dénica motor: file:///C:/Users/frmorquechoan/Downloads/cinematicaydinamicamci-121104092521phpapp02.pdf

Imperial, M. d. (1996). Biblioteca del Motor Diésel. Argentia: Ediciones CEAC, Barcelona.

K. Thriveni, Dr.B.Jaya Chandraiah. (2013). Modeling and Analysis of the Crankshaft Using Ansys Software. IJCER, Vol., 03, 84-89.

Mecánica y Control para Industriales. (31 de 03 de 2010). Recuperado el 11 de 12 de 2017, de Mecánica y Control para Industriales: https://blogs.ua.es/industriales/2010/03/

Norton, R. L. (2009). Diseño De Maquinaria. México: McGraw-Hill.

Sánchez, D. O. (2016). Recuperado el 11 de 12 de 2017, de https://zaguan.unizar.es/record/59100?ln=es

Sánchez, D. O. (2016). Recuperado el 11 de 12 de 2017, de https://zaguan.unizar.es/record/59100/files/TAZ-TFG-2016-2697.pdf

Valdés, J., Coronado, J., \& García, J. (2006). Comparación Y Estudio De La Fractura Del Cigüeñal. Scientia et Technica Año XII, 139-144. 